Case Report

\title{
A Case of Acute Eosinophilic Leukemia with a Novel PHF6 Mutation
}

\author{
J. J. Lipof $\mathbb{D}^{1}{ }^{1}$ E. J. Huselton $\mathbb{D}^{1},{ }^{1}$ C. S. Zent, ${ }^{1}$ A. Evans, ${ }^{2}$ B. Zhang, $^{2}$ P. G. Rothberg, ${ }^{2}$ \\ and J. M. Bennett ${ }^{1,2}$ \\ ${ }^{1}$ Division of Hematology/Oncology, University of Rochester Medical Center, James P. Wilmot Cancer Institute, \\ Rochester, NY, USA \\ ${ }^{2}$ Department of Pathology and Laboratory Medicine, University of Rochester Medical Center, Rochester, NY, USA
}

Correspondence should be addressed to J. J. Lipof; jodi_lipof@urmc.rochester.edu

Received 27 January 2021; Accepted 29 June 2021; Published 5 July 2021

Academic Editor: Tatsuharu Ohno

Copyright (c) 2021 J. J. Lipof et al. This is an open access article distributed under the Creative Commons Attribution License, which permits unrestricted use, distribution, and reproduction in any medium, provided the original work is properly cited.

Acute eosinophilic leukemia (AEL) is a rare form of acute myeloid leukemia (AML) that requires prompt exclusion of reactive etiologies of eosinophilia and identification of an underlying acute myeloid neoplasm. Myeloid neoplasms with prominent eosinophilia often have rearrangements in the platelet-derived growth factor receptor $\alpha$ (PDGFRA) or $\beta$ (PDGFRB) or are associated with core-binding factor AML. In this report, we describe a 35-year-old male presenting with chest discomfort and altered mental status, found to have marked leukocytosis with eosinophilic predominance and an elevated blast count. Bone marrow aspirate and biopsy findings were morphologically consistent with AEL. Fluorescence in situ hybridization (FISH) and standard karyotype analysis did not reveal any abnormalities, and mutation analysis using next generation sequencing (NGS) revealed a pathogenic mutation in PHF6. Cardiac work-up revealed findings suggestive of eosinophilic myocarditis. High-dose glucocorticoid therapy was initiated followed by standard intensive induction chemotherapy with cytarabine and idarubicin. He experienced a rapid reduction in peripheral blood eosinophil and blast count and was found to be in a complete remission at the time of his postinduction bone marrow examination. He underwent allogeneic stem cell transplantation with a matched sibling donor after consolidative high-dose cytarabine and remains in remission at the time of this report, 6 months following his initial diagnosis. The rarity of this condition has resulted in a paucity of data to guide management. Additional studies are needed to better characterize this entity and inform optimal management strategies to attain a long-term sustained remission in these patients.

\section{Introduction}

The differential diagnosis of peripheral blood eosinophilia is broad and includes both malignant and reactive etiologies. Triggers for reactive eosinophilia include drugs, infections (commonly parasitic), and allergies. Patients are diagnosed with hypereosinophilic syndrome (HES) when there is persistent eosinophilia $\left(>1.5 \times 10^{9} / \mathrm{L}\right)$ for greater than 6 months, absence of a clear cause, and clinical manifestations with evidence of organ involvement [1]. Most of these cases are determined to be reactive, resulting from cytokines released in the setting of a known trigger. Rarely, a clonal aberration is identified, and the eosinophilia is found to be related to a myeloproliferative neoplasm (MPN) such as chronic eosinophilic leukemia (CEL), acute myeloid leukemia (AML), or acute lymphoblastic leukemia (ALL). Here, we present a case of acute myeloid leukemia with minimal differentiation and prominent eosinophilia, with a pathogenic mutation involving plant homeodomain finger 6 (PHF6) with a variant allele frequency (VAF) of $13 \%$, as well as a mutation in ZRSR2 (VAF: 73\%).

\section{Case Presentation}

A 35-year-old male with no significant past medical history presented to an outside hospital with fever to $38.9 \mathrm{C}$, altered mental status, headache, and chest pain. EKG showed 
normal sinus rhythm with diffuse ST segment depression in leads II, aVF, and V3-V6. He was found to have leukocytosis with a white blood cell (WBC) count of $55.45 \times 10^{9} / \mathrm{L}$ (normal range: 4.2 to $9.1 \times 10^{9} / \mathrm{L}$ ), thrombocytopenia with a platelet count of $102 \times 10^{9} / \mathrm{L}\left(150\right.$ to $\left.330 \times 10^{9} / \mathrm{L}\right)$, and hemoglobin of $15.7 \mathrm{~g} / \mathrm{dL}(13.7$ to $17.5 \mathrm{~g} / \mathrm{dL})$. The leukocyte differential count revealed marked eosinophilia with an absolute eosinophil count of $16.64 \times 10^{9} / \mathrm{L}\left(0.0\right.$ to $0.5 \times 10^{9}$ / $\mathrm{L})$, representing $30 \%$ of the total $\mathrm{WBC}$ count. The remainder of the differential was significant for $44 \%$ neutrophils, $5 \%$ lymphocytes, $4 \%$ monocytes, $2 \%$ basophils, and $13 \%$ blasts (Figure 1). A transthoracic echocardiogram showed a low normal systolic function with an estimated left ventricular ejection fraction of approximately 55\% and concern for a left ventricular (LV) apical thrombus versus endomyocardial fibrosis. He subsequently underwent cardiac magnetic resonance imaging (MRI) which revealed large areas of subendocardial and midmyocardial delayed enhancement most prominent in the LV apex, suggestive of eosinophilic myocarditis, and confirmed the LV apical thrombus. A left heart catheterization revealed no evidence of coronary artery disease. He was transferred to our institution for further care.

Bone marrow aspirate and biopsy showed acute myeloid leukemia, minimally differentiated, with prominent eosinophilia and $28 \%$ blasts (Figure 2). Flow cytometry on the aspirate specimen revealed a prominent and discrete low side scatter, dim CD45 blast gate comprising approximately $30 \%$ of total events, expressing CD34 (partial, 50\%), CD33, CD38, HLA-DR, CD7 (bright), CD123, and CD71, while negative for cytoplasmic TdT, myeloperoxidase (MPO), and all other lymphoid and monocytic markers (25 additional antigens tested). Bone marrow core biopsy was hypercellular (95\%) with abundant immature mononuclear blast forms and eosinophils present. Immunohistochemistry was negative for CD34, CD117, MPO, TdT, and PAX5.

Chromosome analysis and fluorescence in situ hybridization (FISH) analysis were performed using standard cytogenetic methods by the Cytogenetics Laboratory at the University of Rochester Medical Center. Twenty metaphase spreads were analyzed, and no clonal aberrations were detected. FISH analysis was performed on 200 interphase nuclei and revealed a normal pattern for each of the following probes: PDGFRA (4q12), PDGFRB, BCR/ABL1, $R U N X 1 T 1 / R U N X 1, K M T 2 A$, and $C B F B$. Additional FISH analysis performed at a reference laboratory found no evidence of JAK2 and FGFR1 rearrangements. Taken together, cytogenetic analysis did not detect any recurrent gene rearrangements associated with eosinophilic myeloid neoplasms.

Mutation analysis was performed using next-generation sequencing on the Illumina (San Diego CA) MiSeqDx after library preparation using the Illumina TruSight Myeloid Sequencing Panel. Data analysis was performed using the Illumina MiSeq Reporter and VariantStudio programs, as well as custom software. Analysis revealed a pathogenic mutation in plant homeodomain finger 6 (PFH6) with VAF of $13 \%$. The PHF6 mutation c.684_705delinsCCC causes a shift in the reading frame and premature termination of translation (p.His229ProfsTer44), thus deemed pathogenic as a chain-terminating mutation. Additionally, a mutation was found in ZRSR2 with VAF 73\%. The ZRSR2 mutation c.1332_1343dup is an in-frame duplication of 4 amino acids (p.Ser445_Arg448dup). It has been reported in the gnomAD exomes database at about $0.2 \%$ and is likely not pathogenic.

Therapy was initiated with intravenous methylprednisolone $125 \mathrm{mg}$ daily for 3 days followed by oral prednisone $1 \mathrm{mg} / \mathrm{kg}$ for 4 days. His symptoms improved, and his peripheral blood blast count was reduced to $1 \%$, while the absolute peripheral blood eosinophil count was reduced to $10.6 \times 10^{9} / \mathrm{L}$. This was followed by standard chemotherapy for AML with cytarabine $\left(100 \mathrm{mg} / \mathrm{m}^{2}\right.$ by intravenous continuous infusion on days $1-7)$ and idarubicin $\left(12 \mathrm{mg} / \mathrm{m}^{2}\right.$ intravenously on days $1-3$ ). His day 14 bone marrow biopsy was hypocellular and consistent with chemotherapy-induced ablation, with no morphologic evidence of leukemia. A postinduction biopsy on day 39 was consistent with complete remission with complete hematologic recovery. He had one cycle of consolidative high-dose cytarabine followed by matched sibling donor allogeneic stem cell transplantation. He is in a sustained complete remission at the time of this report, with no evidence of relapse at 6 months after initial diagnosis.

\section{Discussion}

The majority of myeloid neoplasms that present with prominent eosinophilia are associated with rearrangements in the platelet-derived growth factor receptor $\alpha$ (PDGFRA) or $\beta$ (PDGFRB), and fibroblast growth factor receptor 1 (FGFR1), which lead to constitutively active tyrosine kinase fusion genes, and often manifest as MPNs [2]. It is important to assess whether an underlying PDGFR rearrangement is present because even upon transition to a blast phase disease, these can remain sensitive to tyrosine kinase inhibition $[3,4]$. Bone marrow eosinophilia is a feature of core-binding factor AML with $\mathrm{t}(8 ; 21)$ (q22; q22.1) or inv(16) (p13.1q22), which is associated with a favorable prognosis due to a high degree of chemosensitivity [5]. Marrow eosinophilia has also been described in rare forms of myelomonocytic leukemia. ETV6 fusions with different tyrosine kinase proteins including ABL1, FLT3, JAK2, and FGFR3 are associated with leukemogenesis and hypereosinophilia [6]. In these cases, prognosis has largely been determined by the fusion partner and responsiveness to different targeted therapies.

In this report, we describe a patient presenting with acute myeloid leukemia with minimal differentiation and prominent eosinophilia, with a pathogenic mutation in PHF6 at low variant allele frequency. PHF6 is a tumor suppressor gene with its locus on the X-chromosome (Xq26.2). It is recurrently mutated in T-cell acute lymphoblastic lymphoma (ALL) and occurs in about 3\% of all AML cases, much more commonly in males than females [7]. Little is known about the exact mechanisms by which PHF6 mutations may contribute to the pathogenesis of AML or eosinophilia. The majority of these mutations are loss of function mutations, as it was in our case. They are commonly seen without evidence of cytogenetic abnormalities but with 


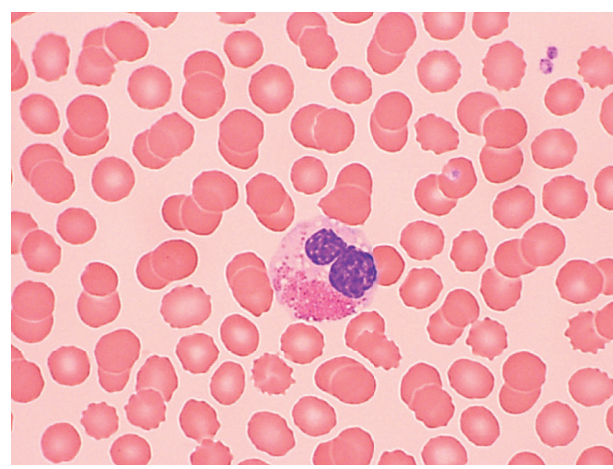

(a)

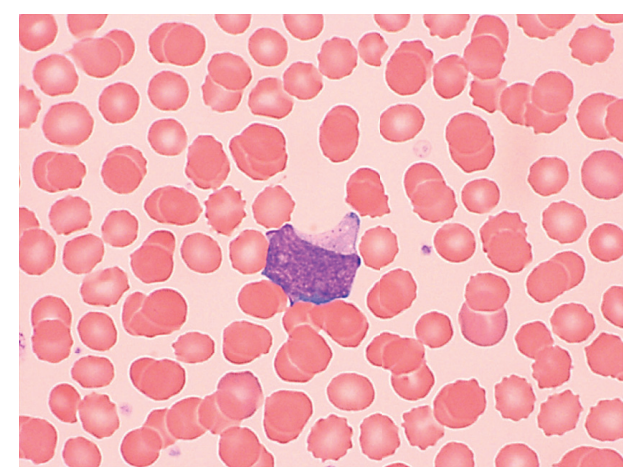

(b)

FIGURE 1: Peripheral blood smear: (a) mature eosinophil with hypogranulation and prominent vacuoles and (b) a blast with rare azurophil granules. Wright-Giemsa stain: 1000x.

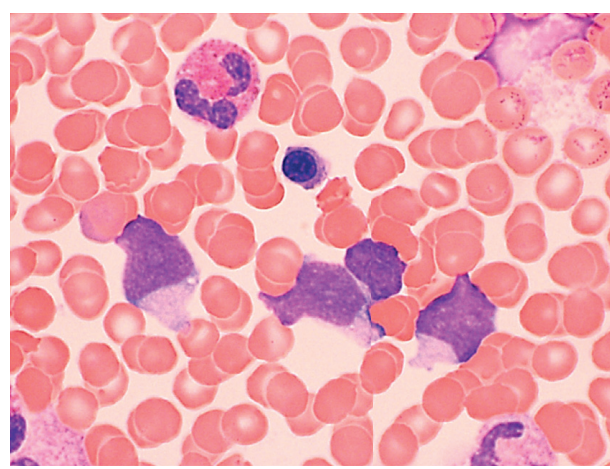

(a)

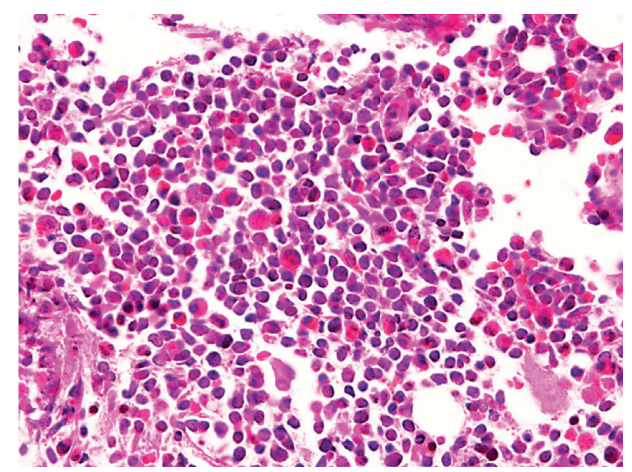

(b)

FiguRE 2: Bone marrow aspirate (BMA) and bone marrow biopsy (BMB): hematoxylin and eosin; 400x: (a) several agranular blasts and (b) numerous eosinophils and immature myeloid precursors.

additional mutations in ASXL1, RUNX1, TET2, and DNMT3A. In this case, the patient had a normal karyotype analysis and a mutation in an additional gene located on the $\mathrm{X}$-chromosome, ZRSR2, which has been detected in $1-3 \%$ of AML cases and has no known prognostic or therapeutic implications $[8,9]$. To our knowledge, this is the first reported case of PHF6-mutated acute myeloid leukemia that presented with prominent eosinophilia, with clinical and radiographic evidence of associated organ involvement. Previous reports have been called either acute eosinophilic leukemia (AEL) or acute myeloid leukemia with eosinophilia $[10,11]$. Eosinophil dysplasia has been noted in both benign and neoplastic disorders but may be helpful in the differential diagnosis of potential myeloid neoplasms [12]. We prefer the term AEL as being consistent with other AML subtypes.

There are limited data to guide the management of these patients due to the rarity of the condition. Our report highlights that, in cases of AEL, it is important to initiate the standard cytogenetic and molecular analysis that is performed in new cases of AML, in addition to evaluation for aberrations that are associated with eosinophilia. In the absence of targetable mutations or chromosomal rearrangements that may inform management, one reasonable approach is the use of high-dose glucocorticoids for symptomatic hypereosinophilia with organ involvement, as well as standard induction chemotherapy followed by allogeneic stem cell transplant for the treatment of AEL. Additional studies are required for better characterization of these patients and determination of the optimal treatment strategies to result in long-term remission.

\section{Conflicts of Interest}

The authors have no conflicts of interest.

\section{Authors' Contributions}

J.J. Lipof drafted the initial manuscript and provided critical revisions. E.J. Huselton and C.S. Zent contributed to data analysis and provided critical revisions. A. Evans contributed to data analysis and interpretation and provided critical revisions. B. Zhang performed chromosome analysis and fluorescence in situ hybridization and provided critical revisions. P.G. Rothberg performed mutational analysis, contributed to data analysis and interpretation, and provided critical revisions. J.M. Bennett contributed to data 
analysis and interpretation, drafted the manuscript, provided critical revisions, and approved the final version.

\section{References}

[1] M. J. Chusid, D. C. Dale, B. C. West, and S. M. Wolff, "The hypereosinophilic syndrome," Medicine, vol. 54, no. 1, pp. 1-27, 1975.

[2] B. J. Bain, "Myeloid and lymphoid neoplasms with eosinophilia and abnormalities of PDGFRA, PDGFRB or FGFR1," Haematologica, vol. 95, no. 5, pp. 696-698, 2010.

[3] P. U. Ogbogu, B. S. Bochner, J. H. Butterfield et al., "Hypereosinophilic syndrome: a multicenter, retrospective analysis of clinical characteristics and response to therapy," Journal of Allergy and Clinical Immunology, vol. 124, no. 6, pp. 1319-1325, 2009.

[4] J. Cools, D. J. DeAngelo, J. Gotlib et al., "A tyrosine kinase created by fusion of thePDGFRAandFIP1L1Genes as a therapeutic target of imatinib in idiopathic hypereosinophilic syndrome," New England Journal of Medicine, vol. 348, no. 13, pp. 1201-1214, 2003.

[5] M. A. Bitter, M. M. Le Beau, R. A. Larson et al., "A morphologic and cytochemical study of acute myelomonocytic leukemia with abnormal marrow eosinophils associated with inv(16)(p13q22)," American Journal of Clinical Pathology, vol. 81, no. 6, pp. 733-741, 1984.

[6] F. Zhou and B. Chen, "Acute myeloid leukemia carrying ETV6 mutations: biologic and clinical features," Hematology, vol. 23, no. 9, pp. 608-612, 2018.

[7] P. Van Vlierberghe, J. Patel, O. Abdel-Wahab et al., "PHF6 mutations in adult acute myeloid leukemia," Leukemia, vol. 25, no. 1, pp. 130-134, 2011.

[8] K. H. Metzeler, T. Herold, M. Rothenberg-Thurley et al., "Spectrum and prognostic relevance of driver gene mutations in acute myeloid leukemia," Blood, vol. 128, no. 5, pp. 686-698, 2016.

[9] F. Thol, S. Kade, C. Schlarmann et al., "Frequency and prognostic impact of mutations in SRSF2, U2AF1, and ZRSR2 in patients with myelodysplastic syndromes," Blood, vol. 119, no. 15 , pp. 3578-3584, 2012.

[10] R. S. Weinger, J. André-Schwartz, J. F. Desforges, and M. Baker, "Acute leukaemia with eosinophilia or acute eosinophilic leukaemia: a dilemma," British Journal of Haematology, vol. 30, no. 1, pp. 65-70, 1975.

[11] M. A. Lichtman and G. B. Segel, "Uncommon phenotypes of acute myelogenous leukemia: basophilic, mast cell, eosinophilic, and myeloid dendritic cell subtypes: a review," Blood Cells, Molecules, and Diseases, vol. 35, no. 3, pp. 370-383, 2005.

[12] J. E. Goasguen, J. M. Bennett, B. J. Bain et al., "The role of eosinophil morphology in distinguishing between reactive eosinophilia and eosinophilia as a feature of a myeloid neoplasm," British Journal of Haematology, vol. 191, no. 3, pp. 497-504, 2020. 